\title{
LA ESTÉTICA EN REVISIÓN: APUNTES CRÍTICOS A LAEMANCIPACIÓN TERRITORIAL EPISTÉMICA DEL CONCEPTO
}

\author{
Aesthetic under Review: Critical Notes on Territorial \\ Epistemic Emancipation of the Concept.
}

María Cristina Ríos Espinosa, Univeridad del Claustro de Sor Juana, México

Correo electrónico: m.riose@universidaddelclaustro.edu.mx

Mayra Sánchez Medina y José Ramón Fabelo Corzo (Coords.), Coordenadas epistemológicas para una estética en construcción. México: Benemérita Universidad Autónoma de Puebla, Instituto de Filosofía de la Habana, 2020, 384 pp.

El libro colectivo Coordenadas epistemológicas para una estética en construcción, coordinado por los investigadores cubanos Mayra Sánchez y José Ramón Fabelo, trata de encontrar nuevas orientaciones acerca del concepto de la estética para emanciparla de su territorialidad epistémica en el saber europeo continental, el cual se presenta con carácter de universal, pero falsea en sí mismo al dejar fuera otros saberes y sensibilidades, invisibilizándolos, como los de los territorios simbólicos americanos considerados premodernos, a pesar de ser simultáneos en el tiempo. El concepto de estética, desde su acuñación semántica por Baumgarten en el siglo XVIII y su teorización por Immanuel Kant y los filósofos románticos alemanes, ha sido caracterizado como una forma de experiencia sensible de un tipo muy especial. La estética se presenta como una facultad depurada de su corporeidad y praxis cotidiana, es decir, una especie de sensibilidad facultativa y teórica a la que Kant llamó "sujeto puro" del entendimiento. Se entiende como el "yo pienso que acompaña todas mis percepciones", de René Descartes, lo cual alude también a la pureza o depuración de los sentimientos. En el fondo, se trata del tipo de subjetividad protestante europea de una civilización triunfante, pero en el campo estético.

El concepto de estética nació en un siglo de grandes convulsiones políticas: el siglo XVIII, dominado por las monarquías absolutas puestas en cuestión; seguido por el XIX, 
tiempos de reconstrucción política en una Europa invadida por las tropas napoleónicas. Fue así como la génesis de su significación perteneció a una época que privilegió las nociones de pureza, buen juicio, genialidad y desinterés por el mundo práctico. El norte de Europa hegemonizó lo estético ligado a un tipo de sensibilidad que correspondió a una historia particular, tiene un fundamento étnico y religioso, que no se ajusta a los modelos de sensibilidad latinoamericanos. Así, las nociones de estética del pasado invisibilizaron estas formas de sensibilidad al considerarlas primitivas, exóticas, premodernas, incivilizadas e inmaduras. Mayra Sánchez y José Ramón Fabelo buscaron de una manera analítica y estimulante para el pensamiento crítico latinoamericano detonar el asidero epistemológico tradicional de la noción de lo estético. En suma, lograron reconstruir el concepto desde una comprensión alternativa de la sensibilidad y sus relaciones en el mundo. Con lo cual se consigue la liberación epistémica bajo una nueva construcción ya desterritorializada de su locus de enunciación teórico original. Además, se sitúa la noción de estética desde el sentir y pensar latinoamericano. En ello consiste la originalidad teórica de las autoras y autores de esta obra colectiva.

La obra está dividida en tres partes que se vinculan entre sí. La primera, "Una Estética en Construcción" incluye seis artículos, los cuales insisten en la necesidad de indagar acerca de nuevas coordenadas epistemológicas en torno a la estética. En ellos se demuestra cómo, desde su nacimiento hasta la era del capitalismo tardío, la noción de estética necesita de una revisión y una deconstrucción, por tratarse de un concepto elitista, colonial y racista, al invisibilizar otros tipos de sensibilidad. Se critica, asimismo, la asociación y reducción de la noción de estética al de lo artístico y del buen gusto. Sánchez, nos invita a hacer una revisión del concepto de estetización del mundo ligada, por lo regular, con el embellecimiento y la estilización.

El primer artículo es una coautoría de Alicia Pino y Mayra Sánchez titulado "Sobre la necesidad de coordenadas epistemológicas". El segundo de esta sección se denomina "La noción de estetización del mundo, una actualización”, por Mayra Sánchez. El tercero, “el cambio cultural como coordenada epistemológica: una reflexión crítica sobre el contexto cultural de la sociedad", de Alicia Pino. El cuarto, "Actualidad en las prácticas simbólicas de arte cubano", de Norma Medero. El quinto, "La territorialidad como coordenada 
epistemológica: problemas e impactos”, por Alicia Pino. El sexto de esta primera sección, "La colonialidad cultural y la lógica del capital” de Jose Ramón Fabelo.

En la segunda parte de este libro, denominada "El arte en el discurso de la estética", se incluyen siete artículos de las y los investigadores del Instituto de Filosofía de la Habana, Cuba, así como del cuerpo académico de la Maestría en Estética y Arte de la Benemérita Universidad Autónoma de Puebla. El primer artículo se titula "Itinerario del arte ante los encerramientos y aperturas de la razón occidental" de Norma Medero; le sigue "Lo concreto y lo complejo en la interpretación del valor del arte”, de José Ramón Fabelo; “La relación arte y ciencia y su impacto en el estatus académico del arte en la universidad", de Mayra Sánchez viene después. Continúan "El arte la ciencia y su relación estética”, por Ramón Patiño, y “Aproximación a las prácticas artísticas de internet su emergencia en Cuba", de Kevin Beovides y Natividad Norma Medero. El apartado cierra con dos contribuciones de José Ramón Fabelo: "La encrucijada axiológica de la reproductibilidad técnica del arte" y "Del arte de Warhol a la interpretación de Danto".

Por último, una tercera parte del libro titulada "Espectacularidad y seducción: Miradas estéticas a la sociedad contemporánea" e incluye siete artículos. El primero, "La sociedad del espectáculo, Debord 50 años después”, de José Ramón Fabelo; el segundo, "La reconfiguración de lo sensible una cuestión estética o política", de Mayra Sánchez; el tercero, "la experiencia contemporánea del vivir", de Gerardo de la Fuente; el cuarto, "Espectáculo versus información: tres miradas", de Alicia Pino; el quinto, "Estetización en Cuba, una mirada deprisa al espacio sensible de los cubanos”, de Mayra Sánchez y Gilberto Valdés. El sexto, "La teatralidad como condición de la socialidad: una mirada desde las escenas norteamericanas de José Martí” de Alicia Pino y Mayra Sánchez, y el séptimo, "Ensanchar el corredor cultural crítico no capitalista en Cuba".

A continuación, dejo algunos apuntes críticos como aperitivo en mi recomendación de lectura. Lo primero que se debe destacar son los aportes de Alicia Pino y Mayra Sánchez en "Sobre la necesidad de coordenadas epistemológicas", quienes tratan de demostrar la manera cómo en el plano cognitivo los conceptos han cambiado su naturaleza y se han quedado vacíos de certidumbres absolutas; éstos ya no pueden ser entendidos desde la certeza, como meros receptáculos de una verdad inamovible, contenedores de todos los problemas. Lo que tratan de mostrar las autoras es cómo estos conceptos, que antes nos 
parecían tan ciertos, ahora no son más que simples axiomas contenedores del saber. Lo que ellas van a intentar hacer es detonarlos, hacerlos explotar en su ilegitimidad, para luego tratar de reconstruirlos sobre nuevas bases epistémicas, pues, si esos conceptos en el pasado servían para una orientación de coordenadas del saber, estaban relacionadas con el poder hegemónico del mismo.

Las autoras insisten en la transversalidad de los saberes y de las disciplinas que hoy no son cerradas o no deben serlo, sino destacar su ser como disciplinas abiertas. De manera crítica nos hacen ver cómo los conceptos en la historia describen un mundo y sus relaciones como si fuesen sustancias estáticas en el tiempo. Esto produce una determinación subjetiva particular, un tipo de sujeto, grupo, territorio, nación y raza, estudiados desde estos paradigmas, se convierten finalmente en obstáculos para poder apreciar toda la riqueza que la vida implica. Por lo tanto, toda la transformación que hemos vivido desde la segunda mitad del 2013 ha sido una revisión obligada de esos conceptos, con la finalidad de descentrarlos de estas miradas normativas e inamovibles: la de un saber hegemónico del mundo occidental que funcionaron como paradigmas del pensamiento y de la crítica, pero cuyas nociones científicas o disciplinares ya no sirven como coordenadas. Las autoras, al citar a la crítica que Michel Foucault hace de los discursos en Arqueología del saber y Microfísica del poder, demuestran cómo a partir del centro de estas teorías existe en su fundamento oculto un saber localizado, o más bien, una localización del saber.

El segundo artículo de la primera sección, escrito por Mayra Sánchez, "La noción estetización del mundo una actualización”, hace una revisión del concepto de estetización y, como bien dice la autora, resultó una novedad en los medios académicos hacia finales del siglo pasado. Hoy en día este concepto aparece como un 'rizoma', una coordenada epistemológica donde podemos estructurar una mirada sagaz de la sociedad contemporánea y sus complejidades. Sánchez critica que el concepto de estetización se haya entendido como embellecimiento, a veces como estilización, ionización, artificación, ligazón o un signo y una multiplicidad de significados, no necesariamente intercambiables. Revisa con rigor particularmente la noción de estetización, de cómo se ha asociado la noción de estética con la belleza, el embellecimiento o engalanamiento. Menciona que los modos de entender lo bello en las diferentes épocas y culturas implicó una correspondencia de las cosas con criterios históricos contextuales de perfección, es decir, la belleza como 
perfección. Pero lo que ella critica de esta noción es que se están acentuando o privilegiando ciertos biotipos y atributos corporales estandarizados occidentales. La autora no está de acuerdo en intercambiar la noción de lo artístico por lo estético, como si la estética se pudiese reducir nada más a la labor artística. Muestra, al final de su ensayo, la forma en que la estética se encuentra en el campo de la cotidianidad y no en el campo de la artisticidad. En este sentido, en tanto que lo estético se reduzca a lo artístico, muchos tipos de sensibilidad son descartados y omitidos. El hecho real de que la estética está en la vida cotidiana nos marca y produce formas de socialización, modos de interacción entre individuos, de tal manera que la estetización es una forma de relacionarnos sensiblemente. Por ello, se le puede ubicar dentro de la cotidianidad y no dentro del embellecimiento del arte o de los museos. Bajo esta última consideración, se anula e invisibiliza la estética como sensibilidad cotidiana, sencilla y corpórea, dejando fuera modos de expresión de las comunidades que se expresan sensiblemente a partir de sus relaciones estéticas.

En la tercera parte del libro, "Espectacularidad y seducción, miradas estéticas a la sociedad contemporánea", el primer capítulo del autor José Ramón Fabelo, "La sociedad del espectáculo de Guy Debord: 50 años después”, analiza la manera como la imagen mediatiza el mundo, el proceso de estetización de la vida. Para el filósofo cubano, la estetización es una noción que desborda el uso de la noción de estética constreñida en la esfera del arte y de lo bello, cuando en realidad está vinculada a lo cotidiano. Reflexiona acerca de cómo la cuestión de la imagen y lo estético adquiere, en el mundo contemporáneo, una significación transartística. Críticamente nos dice que estos cambios en la imagen y lo estético se hacen dentro de los marcos de la lógica del capital, la cual busca manipular, mercantificar y, desde luego, mentir porque no persigue la verdad, sino la maximización de ganancias al costo que sea. ¿Cuál es este costo?, cabría preguntarnos. Desde luego, la destrucción de valores de uso. Sin embargo, Fabelo aporta a la crítica algo adicional: el reconocimiento de la manera en que la estetización del mundo, dentro de la lógica del capital, facilita las relaciones de dominación. Esto ocurre ya que la conquista de los imaginarios sociales invade los modos de simbolización de la gente y se les vende una imagen idealizada de su propia realidad, "se le acostumbra a ver los más profundos dramas humanos como un juego estético" (p. 260). Continúa diciendo que la humanidad se convierte en espectáculo de sí misma y, citando a Benjamin, afirma que "su autoalienación 
[...] le permite vivir su propia destrucción como goce estético" (p. 260). Fabelo nos remite a Guy Debord en "La sociedad del espectáculo", quien es inspirado por esta lapidaria frase de Walter Benjamin: el drama humano se convierte en objeto espectacular de disfrute estético.

Para nuestro crítico cubano, la estetización de la experiencia humana es una colonización del mundo de la vida (Lebenswelt) ${ }^{1}$ por el mass media. Debord, nos dice, lideraba un grupo en los cincuentas llamado situacionista, que dio lugar al movimiento de vanguardia del "Situacionismo francés". Menciona que la explotación capitalista es estetizada como libertad y en el sistema se da la igualación de todos los seres humanos ante la ley. En mi parecer, la originalidad de la contribución de Fabelo reside en desemascarar lo que esta estetización homogenizante oculta, al tratar de considerar las relaciones sociales en el plano de su estetización como unas simétricamente inexistentes en la realidad: aquella que oculta todas las desigualdades sociales en las relaciones de propiedad, anota Fabelo, pero también se aplica al arte popular, a las diferencias étnicas, lingüísticas, devocionales de los pueblos y epistémicas. La asimetría ontológica aparece como simetría. En esto consiste su estetización: una fenomenología de simetría. Fabelo insiste en la manera como la desigualdad en esta sociedad es presentada como igualdad. La injusticia se oculta bajo la apariencia de un orden justo y eso es lo que está en el fondo de la esencia cultural del sistema de la sociedad del espectáculo. Es un reproductor cultural de la desigualdad presentada estéticamente como igualdad, entendiendo, aquí, estéticamente lo que aparece, lo que está en el fondo del sistema. La ontología del sistema es la enajenación, mientras que la enajenación económica es presentada como espectáculo. Por todo ello es estético en el sentido de la representación espectacular de lo simétrico, en el fondo desigual. Para Fabelo, el sistema ha colonizado el ocio, ha banalizado el espíritu y esta es otra vuelta de tuerca a la crítica que hace Marx al hablar de la explotación del trabajo: en este caso se trata de la explotación del ocio, de la colonización de los imaginarios y del tiempo libre de las personas.

En esto consiste la originalidad de los apuntes críticos. El mundo se presenta como espectáculo, como falsificación de la realidad, la ilusión del mundo global de iguales, según

\footnotetext{
${ }^{1}$ El término "Lebenswelt" significa en castellano "Mundo de la vida", es una categoría empleada por el filósofo fenomenólogo Edmund Husserl en Ideas relativas a una fenomenología pura y una filosofía fenomenológica, (México, Fondo de Cultura Económica, trad. José Gaos, 1986), 17-18.
} 
José Ramón Fabelo. En una argumentación interesante, nuestro autor señala que, si intentamos cerrar las puertas del espectáculo, la lógica del capitalismo, el poder hegemónico mundial obliga a abrirlas, y por lo tanto procrea nuevos espectáculos.

Invito al lector a revisar estas interesantes contribuciones en la teoría estética contemporánea. 